\title{
Childhood Trauma and Neural Responses to Personalized Stress, Favorite-Food and Neutral-Relaxing Cues in Adolescents
}

\author{
James Elsey ${ }^{1,2}$, Alice Coates ${ }^{1,2}$, Cheryl M Lacadie ${ }^{3}$, Eamon J McCrory ${ }^{4}$, Rajita Sinha ${ }^{5,6}$, Linda C Mayes ${ }^{1,2}$ and \\ Marc N Potenza*,1,5,6,7 \\ 'Child Study Center, Yale University, New Haven, CT, USA; ${ }^{2}$ Anna Freud Centre, University College London, London, UK; ${ }^{3}$ Department of \\ Diagnostic Radiology, Yale University, New Haven, CT, USA; ${ }^{2}$ Division of Psychology and Language Sciences, University College London, London, \\ UK; ${ }^{5}$ Department of Psychiatry, Yale University, New Haven, CT, USA; ${ }^{6}$ Department of Neurobiology, Yale University, New Haven, CT, USA; \\ ${ }^{7}$ Connecticut Mental Health Center, New Haven, CT, USA
}

Previous studies have found childhood trauma to be associated with functional and structural abnormalities in corticostriatal-limbic brain regions, which may explain the associations between trauma and negative mental and physical health outcomes. However, functional neuroimaging of maltreatment-related trauma has been limited by largely using generic and predominantly aversive stimuli. Personalized stress, favorite-food, and neutral/relaxing cues during functional magnetic resonance imaging were used to probe the neural correlates of emotional/motivational states in adolescents with varying exposure to maltreatment-related trauma. Sixty-four adolescents were stratified into high- or low-trauma-exposed groups. Cue-related measures of subjective anxiety and craving were collected. Relative to the low-trauma-exposed group, high-trauma-exposed adolescents displayed an increased activation of insula, anterior cingulate, and prefrontal cortex in response to stress cues. Activation in subcortical structures, including the hippocampus, was inversely correlated with subjective anxiety in the high- but not the low-trauma-exposed group. The high-trauma-exposed group displayed hypoactivity of cerebellar regions in response to neutral/relaxing cues. No group differences were observed in response to favorite-food cues. The relationship between trauma exposure and altered cortico-limbic circuitry may in part explain the association between childhood trauma and heightened vulnerability to emotional disturbances and risky behaviour. This may be particularly pertinent during adolescence when such difficulties often emerge. Further work is needed to elucidate the mechanism linking trauma to obesity.

Neuropsychopharmacology (2015) 40, I580-1589; doi:I0.1038/npp.20I5.6; published online I8 February 2015

\section{INTRODUCTION}

Childhood trauma is associated with multiple psychiatric disorders (eg, anxiety and depression) and other health complications (eg, obesity, risky sexual behaviour, and substance use) (Cohen et al, 2001; Gilbert et al, 2009). Adolescence is an important epoch for the development of affective neurocircuitry (Somerville et al, 2010) and is a time when many risk behaviors and psychiatric disturbances begin to emerge (Paus et al, 2008). Investigating the neural responses of adolescents to emotional and motivational stimuli may provide an insight into the trajectories relating to early adversity.

Early adverse experiences, including maltreatment, may sensitize the hypothalamic-pituitary-adrenal (HPA) axis

* Correspondence: Professor MN Potenza, Yale University School of Medicine, Departments of Psychiatry, Child Study, and Neurobiology, 34 Park Street, New Haven, CT, 065 19, USA, Tel: + I 203737 3553, Fax: + 203737 359I, E-mail: marc.potenza@yale.edu Received 21 July 2014; revised 30 December 2014; accepted 30 December 2014; accepted article preview online 8 January 2015
(Heim and Nemeroff, 2001), possibly causing neurotoxic effects, particularly in glucocorticoid-receptor-dense regions. Structural and functional alterations in relation to childhood trauma, chronic or cumulative stress have been observed in regions related to autonomic and emotion processing and regulation, including the medial prefrontal cortex (MPFC), amygdala, hippocampus, insula, and cerebellum (Dannlowski et al, 2012; Edmiston et al, 2011; Seo et al, 2014).

Existing neuroimaging research of childhood trauma has typically used generic (not personalized) stimuli. Such studies are not able to examine brain function underlying the processing of personally salient information (Hommer et al, 2013). Research has also focused primarily on aversive states, although maltreatment could be related to changes in the processing of appetitive and relaxed states as well. For example, life history theories indicate that organisms adapt their developmental trajectories to their context (Jeffry \& Belsky, 2008). A potentially adaptive neurobehavioral phenotype of hypervigilance and stress reactivity (McCrory et al, 2011; Pollak, 2008) following maltreatment could also result in differential propensities to 
experience relaxing states. Additionally, in adverse rearing environments, appetitive circuitry might be changed on account of the unpredictability of resources, eliciting enhanced salience of appetitive cues and perhaps leading to maltreatment's association with obesity (Bentley and Widom, 2009; Thomas et al, 2008). Childhood trauma might therefore impact upon a broader range of emotional and motivational states than have been previously investigated.

To address gaps in the current understanding of the correlates of maltreatment-related trauma, functional magnetic resonance imaging (fMRI) was used to assess regional brain activations to personalized appetitive (favorite food), aversive (stress), and neutral/relaxing cues in adolescents segregated into high- or low-trauma-exposed groups. The guided-imagery fMRI procedure, in which personal experiences are recalled and developed into scripts to be recorded and played back in the scanner, is validated and has been used in both adults and adolescents (Hommer et al, 2013; Jastreboff et al, 2013; Sinha, 2013). To our knowledge, this is the first study to investigate the relationship between childhood trauma and the neural correlates of personally salient appetitive, aversive, and neutral/relaxing cues.

Based upon previous research (Hommer et al, 2013; Seo et al, 2014), we hypothesized a condition-by-trauma interaction with the following predictions.

\section{Group Comparisons}

Stress cues. In light of past studies of childhood and cumulative trauma, it was predicted that increased activation of cortico-limbic regions (eg, lateral prefrontal cortex, anterior cingulate, amygdala, and insula) to stress cues in the high-trauma-exposed relative to the low-trauma-exposed group would be observed (McCrory et al, 2011; Seo et al, 2014).

Neutral/relaxing cues. The cerebellum and anterior cingulate cortex (ACC) have been implicated in relaxation and appear sensitive to stress-related neurotoxic effects (Critchley et al, 2001). These regions were predicted to display reduced activation in the high-trauma-exposed relative to low-trauma-exposed group in response to neutral-relaxing cues as a possible consequence of such effects.

Favorite-food cues. It was predicted that the high-trauma group would display a reduced activation in prefrontal cortical regulatory regions in response to the food cues. This was based upon the association between early maltreatment and factors linked to dysregulated eating behaviour (Bentley and Widom, 2009), which might suggest reduced activation of brain regions involved in regulating appetitive drives.

Correlations between subjective ratings and brain activation. The findings were expected to replicate the previously reported inverse correlations between corticostriatal-limbic activity and subjective anxiety in response to stress cues (Hommer et al, 2013; Seo et al, 2014). Replication of the previously observed inverse association between cortico-striatal activity and food craving in response to favorite-food cues was also anticipated (Hommer et al, 2013; Seo et al, 2014). We aimed to explore whether childhood trauma might modulate these relationships.

\section{MATERIALS AND METHODS}

Sixty-seven adolescents were recruited for fMRI from an ongoing longitudinal study of disadvantaged youth (Mayes et al, 2005) who had been enrolled at birth and followed every 6 months thereafter. Three adolescents were excluded for technical reasons. The final sample consisted of 64 adolescents ( 40 boys, 24 girls) between 14 and 18 years of age $(M=15.39, \mathrm{SD}=0.89)$. The sample was predominantly African-American (79.7\%), with a minority of participants being Caucasian (9.4\%) or of other ethnicity $(10.9 \%)$. IQ $(M=91.52, \mathrm{SD}=12.16$, range $=68.20-119.60)$ was determined using the Kaufman Assessment Battery for Children composite score (KABC) (Kaufman and Kaufman, 1983).

Forty-four $(68.75 \%)$ of the adolescents had been prenatally exposed to cocaine, as determined by urine screens at prenatal visits or delivery and self-reported use during pregnancy. Recent stress was assessed using the 10-item Perceived Stress Scale $(M=10.83, \quad S D=3.78) \quad(P S S-10)$ (Cohen, 1988), a retrospective self-report measure determining subjective experience of stress within the previous 30 days and appropriate for use in adolescents. Exclusion criteria were significant medical or mental illness, use of psychotropic medications or medications that might influence autonomic responses, non-removable metal in the body, and inability to be comfortable in the MRI machine because of body size or claustrophobia. The National Institute of Mental Health Diagnostic Interview Schedule for Children (NIMH DISC-IV) (Shaffer et al, 2000) was used to determine the mental health. No participants met criteria for any Axis 1 disorder.

Childhood trauma was assessed using the Childhood Trauma Questionnaire Short-Form (CTQ-SF) (Bernstein and Fink, 1998), a well validated and reliable retrospective self-report measure of abuse and neglect before the age of 18 (Bernstein et al, 1997; Bernstein and Fink, 1998; Fink et al, 1995; Paivio and Cramer, 2004; Scher et al, 2001). A summary score for maltreatment was calculated by summing the scores for all 25 clinical items (Scher et al, 2001). The mean score was $35.67(\mathrm{SD}=9.94)$. This score indicates marginally above average exposure to childhood trauma (Scher et al, 2001), consistent with disadvantaged groups who represent an at-risk population for exposure to adverse life events (Mayes et al, 2005). High ( $\geqslant 34, n=31$ ) and low $(<34, n=33)$ maltreatment groups were defined by median split. All procedures were approved by the Yale Human Investigation Committee and written informed consent received from the parent (with participant assent) or participant.

\section{Imagery Script Development}

The personalized guided-imagery fMRI procedure (described in detail in the Supplementary Materials; see also Sinha, 2009; Sinha, 2013; and Sinha \& Tuit, 2012) has 
been used previously to elicit anxiety, food craving, and relaxation through the vivid remembrance of stressful, craving-related, and neutral/relaxing scenarios (Hommer et al, 2013; Jastreboff et al, 2013; Seo et al, 2014). Two scripts each were developed for stress, favorite-food, and neutral/relaxing conditions using the Scene Development Questionnaire (Sinha, 2013) and audiotaped in a female voice for presentation in the scanner. Examples of stress, favorite-food, and neutral/relaxing scripts are provided elsewhere (Yip et al, 2014).

\section{fMRI Procedure}

Participants familiarized themselves with the setting in a mock scanner. For data collection, the six scripts were presented through headphones in a female voice in randomized, counterbalanced order. Each trial consisted of $1.5 \mathrm{~min}$ quiet baseline, $2.5 \mathrm{~min}$ imagery, and $1 \mathrm{~min}$ recovery periods. Immediately before and after each trial, participants rated their feelings of anxiety and food craving on $0-10$ scales $(0=$ not at all, $10=$ more than ever $)$. To preclude carryover effects, a 2 min progressive relaxation period followed each trial and the next trial was not initiated until subjective ratings of craving and anxiety were at the preceding trial's baseline level.

\section{Image Acquisition}

Image acquisition was conducted with a 3T Siemens Trio MRI system, including a standard quadrature head coil and using a T2*-sensitive gradient-recalled single-shot echoplanar pulse sequence. Spin-echo imaging in the axial plane parallel to the anterior-commissure/posterior-commissure $(\mathrm{AC} / \mathrm{PC})$ line with repetition time $(\mathrm{TR})=300 \mathrm{~ms}$, echo time $(\mathrm{TE})=2.46 \mathrm{~ms}$, bandwidth $=310 \mathrm{~Hz} /$ pixel, flip angle $=60^{\circ}$, field of view $(\mathrm{FOV})=220 \times 220 \mathrm{~mm}$, matrix $=256 \times 256,32$ slices with slice thickness $=4 \mathrm{~mm}$ (no gap) was employed to obtain anatomical images of the functional slice locations. For functional image acquisition, a single-shot gradient echo-planar-imaging sequence was used. Thirty-two axial slices parallel to the AC/PC line covering the whole brain were acquired with $\mathrm{TR}=2000 \mathrm{~ms}, \mathrm{TE}=25 \mathrm{~ms}$, bandwidth $=2005 \mathrm{~Hz} /$ pixel, flip angle $=85^{\circ}, \quad F O V=220 \times 220$ $\mathrm{mm}$, matrix $=64 \times 64,32$ slices with slice thickness $=4 \mathrm{~mm}$ (no gap, 150 measurements. After functional imaging, a high-resolution 3D magnetization-prepared-rapid-gradientecho (MPRAGE) sequence $(\mathrm{TR}=2530 \mathrm{~ms}$; $\mathrm{TE}=3.34 \mathrm{~ms}$; bandwidth $=180 \mathrm{~Hz} /$ pixel; flip angle $=7^{\circ}$; slice thickness $=$ $1 \mathrm{~mm} ; \mathrm{FOV}=256 \times 256 \mathrm{~mm}$; matrix $=256 \times 256$ ) was used to get sagittal images for multi-subject registration.

\section{fMRI Analysis}

XMedCon (Nolf et al, 2003) was used to convert data from Digital Imaging and Communication in Medicine (DICOM) format to analyze format. During this process, the first ten images beginning each of the six functional series were discarded to allow the signal to achieve steady-state equilibrium, leaving 140 measurements for analysis. Motion correction for three translational and three rotational directions was performed in SPM5 (www.fil.ion.ucl.ac.uk/spm) and trials with linear motion $>1.5 \mathrm{~mm}$ or rotation $>2^{\circ}$ were discarded. A general linear model on each voxel in the entire brain volume with a task-specific regressor was used to analyze individual subject data. The regressor was the time-block during which participants listened to the guidedimagery script, compared with the baseline resting period. The recovery period $(1 \mathrm{~min})$ was excluded from the data analysis to prevent carryover effects from the imagery period. For temporal filtering, a drift correction was included in the general linear model. Drift regressors were used to remove the mean time course, linear trend, quadratic trend, and cubic trend for each run. Functional images from scripts of the same type were averaged and spatially smoothed with a 6-mm Gaussian kernel. The output maps were normalized beta maps, in the acquired space $(3.44 \times 3.44 \times 4 \mathrm{~mm})$.

BioImage Suite software (http://www.bioimagesuite.org/) (Duncan et al, 2004) was used to concatenate three registrations to apply as one registration to the individual normalized beta maps, bringing the data into a common reference space. First, linear registration was conducted to register each subject's raw functional images with their respective $2 \mathrm{D}$ anatomical images, and then again to register these $2 \mathrm{D}$ images with their $3 \mathrm{D}$ anatomical image. A nonlinear registration was then computed between the individual 3D anatomical image and a reference 3D image, the Colin27 Brain (Holmes et al, 1998), in Montreal Neurological Institute (MNI) space.

Data were converted to AFNI format (http://afni.nimh. nih.gov) (Cox, 1996) for second-level group analysis with random mixed-effects models. Data were returned to ANALYZE format for viewing in BioImage Suite. Effects of condition (stress vs neutral/relaxing vs favorite-foodcraving) and trauma (high vs low) were examined in AFNI Matlab library in a two-way ANOVA with subject treated as a random factor. Family-wise-error (FWE) correction for multiple comparisons (Cox, 1996; Xiong et al, 1995) was performed by using the Monte Carlo simulations with AlphaSim. The source of significant effects was determined by using the whole brain, condition-specific contrasts and trauma contrasts.

Biolmage suite was used to perform whole-brain correlational analyses. Post-imagery anxiety and craving measures for the stress and food-craving conditions, respectively, were correlated with brain activation measures, with the application of FWE-correction for multiple comparisons. Biolmage Suite was used to perform Fisher's Z-transformation (Fisher, 1921) to compare the relationships between bold response and subjective anxiety and craving between high- and low-trauma-exposure groups, with whole-brain (FWE) correction. To plot associations between regional brain activations and subjective measures that differed across groups, beta values were extracted from the regions identified as displaying significantly different correlations between groups. The beta values were extracted for each participant for the respective condition using BioImage Suite. These beta values were plotted against subjective ratings within each group, using IBM SPSS to generate $r$ values. Significance was tested with and without removal of outlying beta values. Graphs and statistics are presented after outlier removal; the pattern and significance of results were comparable before outlier removal. 


\section{RESULTS}

\section{High and Low-Trauma-Exposed Group Demographic Information}

Mean and standard deviations scores for participant information in each group, as well as test statistics for group comparisons, are displayed in Table 1. According to normative data (Scher et al, 2001), CTQ values place the average high-trauma-exposed group participant in the 90th percentile for traumatic experiences, and the average lowtrauma-exposed group participant between the 25th and 50th percentiles, indicating that the median split succeeded in producing groups reflecting high and low levels of childhood trauma.

The distribution of gender, ethnicity, and scanning procedure were comparable between groups (all $p>0.1$ ). Age, IQ and perceived stress were also equivalent between groups (all $p>0.1$ ). Five participants were unable to attend sessions to determine IQ, but there was no evidence of systematic differences in attendance between groups $(p>0.1)$. Prenatal cocaine exposure was over-represented in the high- relative to the low-trauma-exposed group $\left(\chi^{2}(1)=3.96, p<0.05\right)$ (Supplementary Figures S1, S2, S3 \& S4 in Supplementary Materials show inclusion of prenatal cocaine exposure as a factor of no interest into the ANOVA had no/minimal influence on findings).

\section{Subjective Responses to fMRI Trials}

Participants reported subjective anxiety and food craving $(0-10)$ immediately before and after each fMRI condition trial (Figure 1). Anxiety increased following stress trials $(t(62)=3.70, p<0.001$; baseline $M=3.43, \mathrm{SD}=2.39$; postimagery $M=3.86, \mathrm{SD}=2.53$ ) but not after neutral/relaxing trials $(t(62)=1.39, p=0.17$; baseline $M=3.40, \mathrm{SD}=2.43$; post-imagery $M=3.55, \mathrm{SD}=2.53)$. Food craving increased following favorite-food trials $(t=6.21, p<0.001$; baseline $M=4.69, \mathrm{SD}=2.89$; post-imagery $\mathrm{M}=5.82, \mathrm{SD}=2.93)$ but not after neutral/relaxing trials $(t=0.22, p=0.83$; baseline $M=5.10, \quad \mathrm{SD}=3.09$; post-imagery $\mathrm{M}=5.13, \mathrm{SD}=2.94$ ). Compared with neutral/relaxing trials, stress trials induced a greater anxiety $(t(62)=1.87, p<0.05)$, and favorite-food trials induced a greater food craving $(t(62)=4.25, p<0.001)$.
The trauma groups reported similar imagery-induced changes in subjective ratings and baseline and post-imagery ratings of anxiety and food craving (all $p>0.1$ ). These results indicate that stressful and appetitive imagery conditions successfully elicited the anticipated increases in anxiety and food craving across groups, respectively.

\section{fMRI Measures}

All analyses were thresholded at $p<0.05$ two-tailed, wholebrain FWE-corrected.

\section{Main Effects and Interactions}

Analyses yielded five significant activation clusters for the main effect of condition, comprising cortical (middle/

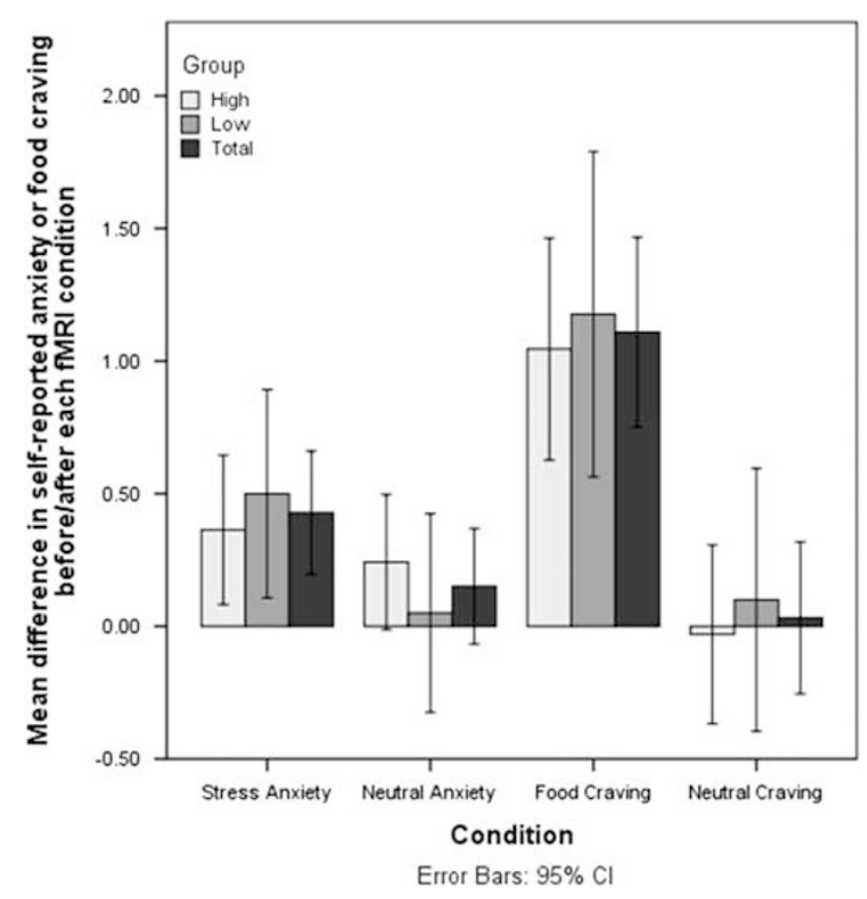

Figure I Subjective reports of anxiety and food craving in response to stress, favorite-food, and neutral/relaxing cues.

Table I Group Demographic Information and Group Comparisons

Trauma-exposure Group

\begin{tabular}{|c|c|c|c|c|}
\hline & \\
\hline & Low M(SD) & High M(SD) & $t / \chi^{2}$ & $P$ \\
\hline Age & $15.25(0.75)$ & I5.55 (1.01) & $t(62)=1.339$ & $p=0.185$ \\
\hline Gender (male/female) & $19 / 14$ & $21 / 10$ & $\chi^{2}(1)=0.705$ & $p=0.401$ \\
\hline CTQ total & $28.36(2.80)$ & $43.45(8.82)$ & $t(62)=9.345$ & $p<0.00$ \\
\hline PSS & $10.33(3.89)$ & $11.35(3.65)$ & $t(62)=1.081$ & $p=0.284$ \\
\hline
\end{tabular}

Abbreviations: AA, African-American; C, Caucasian; CTQ, childhood trauma questionnaire short-form; KABC, Kaufman assessment battery for children; M, mean; $\mathrm{N}$, no, not exposed to cocaine prenatally; $\mathrm{O}$, other; PSS, perceived stress scale; SD, standard deviation; $t=$ independent samples $t$-test; $\chi^{2}=$ Pearson's Chi square goodness of fit test; $Y$, yes, exposed to cocaine prenatally. 
superior temporal gyrus, insula, and PFC) and subcortical (cerebellum, thalamus, caudate, and midbrain) brain regions (Supplementary Figure S5, Supplementary Table S1). There was no main effect of trauma but a significant trauma-group-by-condition interaction, composed of three activation clusters comprising predominantly cortical brain regions including the caudal anterior cingulate and posterior cingulate, insula, ventrolateral prefrontal cortex, visual cortex, superior/middle temporal gyrus, premotor, primary motor and sensory cortices, fusiform, and cerebellum (Supplementary Figure S6, Supplementary Table S2, see Supplementary Figure S7 and Supplementary Materials for further analysis of the interaction).

\section{Brain Responses of High- vs Low-Trauma-Exposed Groups to Stress Cues}

Contrasting high- and low-trauma-exposed groups in the stress condition revealed three principal clusters in which the high-trauma-exposed group displayed greater activation than the low-trauma-exposed group (Figure 2, Supplementary Table S3). The high- vs low-trauma-exposed group displayed increased responsivity in cortical regions including the insula, caudal ACC, premotor cortex, primary motor, visual and sensory cortices, middle/superior temporal gyrus, fusiform, and occipitoparietal regions (BA7, BA19) bilaterally, in addition to right rostral anterior cingulate and medial/lateral prefrontal cortex activation.

\section{Relationships Between Brain Responses and Anxiety in Response to Stress Cues}

Correlational analyses across all participants revealed anxiety to be inversely correlated with activity in multiple corticostriatal-limbic regions (Supplementary Figure S8, Supplementary Table S4), notably in the thalamus, ventral striatum, midbrain, hippocampus/parahippocampus, amygdala, and prefrontal cortex. Significant correlations were also observed in premotor and primary motor cortex, middle/superior temporal gyrus, cerebellum, and primary sensory cortex extending into occipitoparietal regions.

Analysis of differences between trauma-exposed groups in these correlations showed that activation in the left hippocampus/parahippocampus (high $r(27)=-0.402$, $p=<0.05$, low $r(30)=0.197, p>0.1$, Figure $4 \mathrm{~b})$, posterior caudate (high $r(27)=-0.465, p<0.01$, low $r(31)=0.195$, $p>0.1$ ), posterior insula (high $r(26)=-0.659, p<0.001$, low $r(29)=0.007, p>0.1$ ), thalamus (high $r(25)=-0.500$, $p=<0.01$, low $r(29)=0.217 p>0.1)$ temporal pole (High $r(26)=-0.564, p<0.005$, low $r(31)=0.183, p>0.1)$, and midbrain (high $r(29)=-0.553, p<0.005$, low $r(32)=0.165$, $p>0.1)$ had significantly different relationships to postimagery anxiety in the high- and low-trauma-exposed groups. In these regions, activation was inversely correlated with post-imagery anxiety only in the high-trauma-exposed group (Figure 3a, Supplementary Table S5).

\section{Brain Responses of High- vs Low-Trauma-Exposed Groups to Neutral/Relaxing Cues}

Comparisons between the high- and low-trauma-exposed groups revealed significantly decreased activation in the cerebellar vermis and right cerebellum in the high-traumaexposed group relative to the low-trauma-exposed group (Figure 4, Supplementary Table S6).

\section{Brain Responses of High- vs Low-Trauma-Exposed to Favorite-Food Cues}

Comparing favorite-food-related brain activations in the highand low-trauma-exposed groups did not reveal between-group differences surviving the whole-brain correction.

\section{Relationship Between Brain Responses and Craving in Response to Favorite-Food Cues}

An inverse correlation between food craving and corticostriatal activation, specifically in the left caudate and

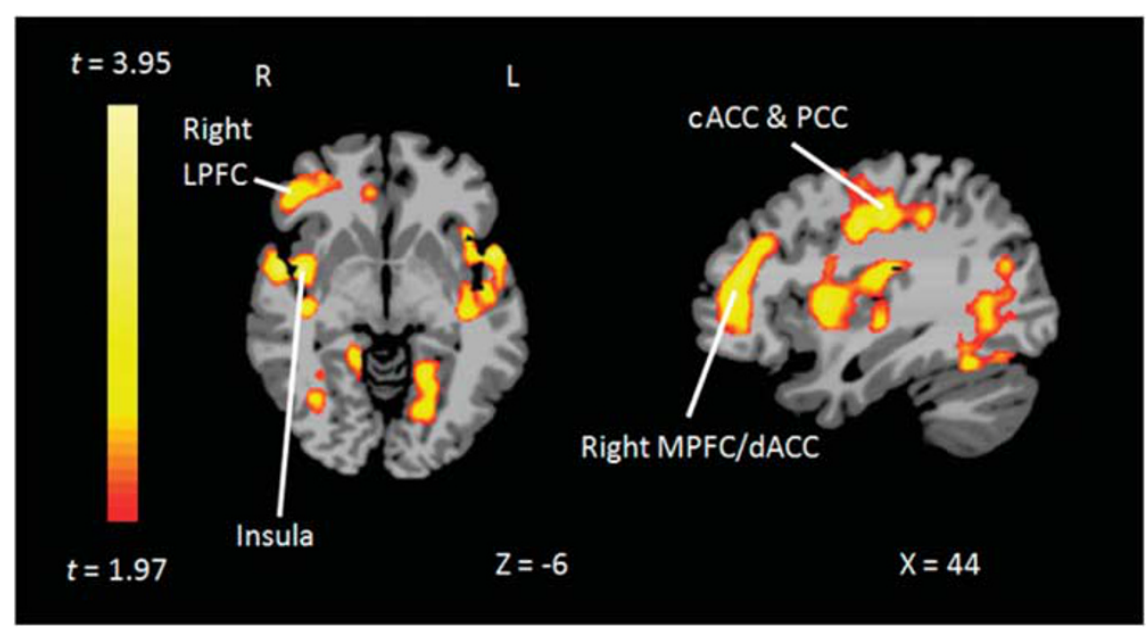

Figure 2 High- vs low-trauma-exposed group contrast for stress cues. Brain regions showing a significantly greater activation in the high-trauma-exposed group in response to stress cues include the insula, right lateral prefrontal cortex (LPFC), right medial prefrontal cortex (MPFC) and dorsal ACC (dACC) caudal ACC (cACC), and the posterior cingulate cortex (PCC). Analyses were thresholded at $p<0.05$ two-tailed, whole-brain FWE-corrected. Coordinates are in $M N I$ space. $R=$ right, $L=$ left. 
a

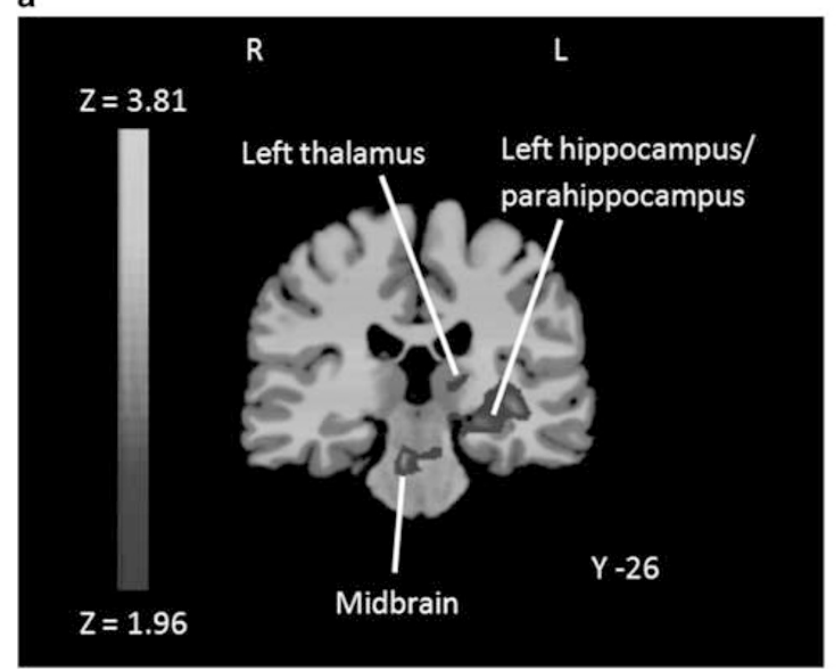

b

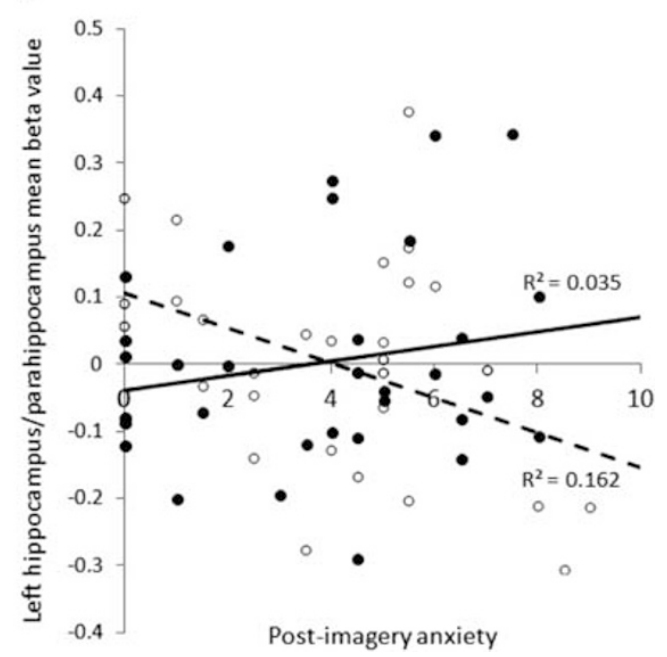

Figure 3 (a) Regions showing significantly different correlations between post-imagery anxiety and BOLD responses to stress cues between high- and low-trauma-exposed group, including the midbrain, left thalamus, and left hippocampus/parahippocampus. Blue/violet voxels indicate a significantly more negative correlation in the high relative to the low adversity group. (b) Scatter plot depicts the association between brain activity and post-imagery anxiety in the high- (dashed line, open dot) and low- (solid line, solid dot) trauma-exposed group groups for the hippocampus/parahippocampus, with associated $R^{2}$ values. The interaction reflects differences in the correlation with a negative slope in the high-trauma-exposed group and the correlation with a positive slope in the low-trauma-exposed group. Analyses were thresholded at $p<0.05$ two-tailed, whole-brain FWE-corrected. Coordinates are in MNI space. $R=$ right, $L=$ left.

prefrontal cortex, was found in the favoritie-food condition (Supplementary Figure S9, Supplementary Table S7). Inverse associations were also seen in primary visual and visual association cortex, extending into occipitoparietal regions. Analysis of between-group differences in these correlations failed to show regions with significantly different brain activation-craving associations between groups.

\section{DISCUSSION}

This study is the first to assess the relationship between childhood trauma and neural responses to stress, favoritefood, and neutral/relaxing cues in a group of adolescents. Moreover, by utilizing individualized and complex cues, the paradigm used a more closely approximated real-life affective situations and provided insight into the processing of personally salient events compared with previous studies. The present findings suggest that childhood trauma leads to neural hyper-responsivity to acute stress in prefrontal regulatory regions as well as the insula. Hypoactivation of subcortical structures, notably the hippocampus, was associated with anxiety in the response to stress cues, specifically in the high-trauma-exposed group. Trauma exposure was also linked to hypoactivation of cerebellar regions in response to the neutral/relaxing cues. These differences were observed in the absence of contemporaneous psychiatric disturbances.

Regions showing hyper-responsivity to stress cues, notably the lateral and medial prefrontal cortex and ACC, have important roles in the control of limbic neurocircuitry and emotional regulation (Ochsner et al, 2002). Insula activation has been related to the processing of aversive

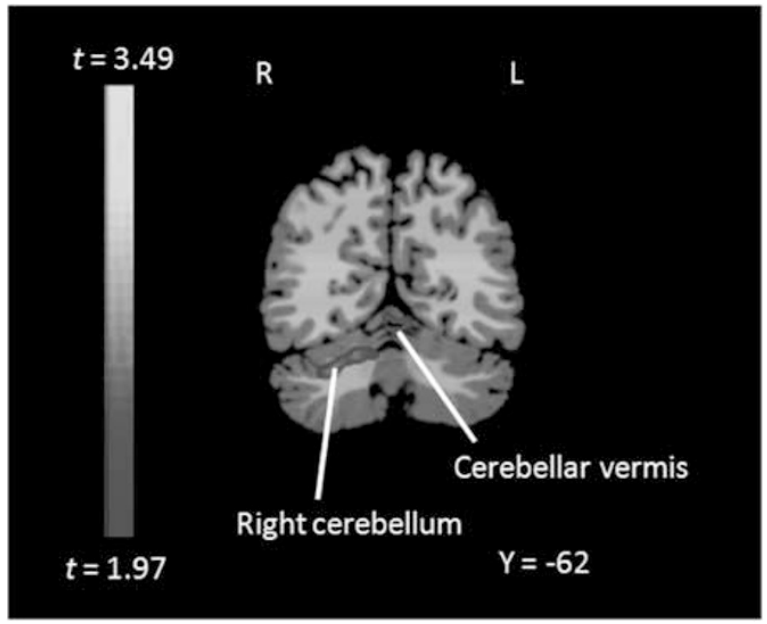

Figure 4 High- vs low-trauma-exposed group contrast for neutral/ relaxing cues. Brain regions showing significantly lower activation in the high-trauma-exposed group in response to neutral/relaxing cues were the cerebellar vermis and right cerebellum. Analyses were thresholded at $p<0.05$ two-tailed, whole-brain FWE-corrected. Coordinates are in $\mathrm{MNI}$ space. $R=$ right, $L=$ left.

cues and interoceptive states (Critchley et al, 2004) and hyper-responsivity of the insula has been reported in trauma-exposed children during the processing of angry faces (McCrory et al, 2011). More generally, hyperresponsivity to stress cues in these regions has been reported in adults as a function of cumulative adversity across the lifespan (Seo et al, 2014). Structurally, differences in cortical thickness and local gyrification have been observed in trauma-exposed children in these regions 
(Kelly et al, 2013); in adults, reduced gray-matter densities in these regions have been associated with childhood trauma and lifetime adversity (Edmiston et al, 2011). This study extends these findings by demonstrating functional changes in these regions in association with childhood trauma in response to personally relevant cues. Heightened activation of these regions in the high-trauma-exposed group is consistent with heightened neural stress reactivity in this group (Loman and Gunnar, 2010), which may require compensatory increases in prefrontal regulatory activity to maintain stress responses within normal limits. This may partly explain why higher anxiety was related to reduced prefrontal activity in the stress condition. Notably, such differences between trauma groups were observed in the absence of psychiatric disorder. As such, they may represent neural biomarkers of vulnerability, which may aid the identification of those at risk of disorder and allow for early intervention.

Interestingly, while increased regional activation was observed in the high- relative to low-trauma groups, an inverse correlation between subjective anxiety and regional activations (eg, in hippocampus, thalamus, and midbrain) was observed in the high-trauma group. These findings suggest that subjective experiencing of stress relates to regional brain activations in a fashion in which there may exist a 'blunting' of subjective responses in relation to the activation patterns of brain regions processing stress in the high-trauma group. Such a relationship might reflect neuroadaptive processes relating to repeated exposure to stress or trauma. However, this interpretation is speculative and warrants an additional investigation.

Hyper-responsivity of the amygdala found in previous studies linking maltreatment-related trauma to neural responses to aversive cues (McCrory et al, 2011) was not observed in the present study. McCrory et al (McCrory et al, 2011) used the standardized facial stimuli that may activate the amygdala given its function in detecting salient stimuli (Sander et al, 2003). The present study focused on imagining complex and personally relevant autobiographical scenarios, more likely to elicit the amygdala functioning in relation to the emotional memory (Dolcos et al, 2005). This suggests that the impact of trauma on brain function may be context/task-dependent.

Differences in the relationships between neural activity and anxiety during stress imagery indicate that while subjective anxiety may have been comparable between groups, the neural processing of anxiety could differ as a function of trauma exposure. Of note is the relationship between hippocampal hypoactivation and heightened anxiety in the high-trauma-exposed group. The hippocampus is sensitive to neurotoxic glucocorticoid effects and glucocorticoid levels are increased in individuals with a history of trauma (Anderson et al, 2002; Heim and Nemeroff, 2001), but it has been suggested that morphological changes in this region in relation to trauma may not appear until later life (Woon and Hedges, 2008). The present findings demonstrate that differential functionality of this region during stress may arise as early as adolescence. The hippocampus is a crucial brain region for the integration of explicit autobiographical memories. Differential functioning of the hippocampus in adolescence is consistent with over-general autobiographical memory processing (in which individuals struggle to retrieve specific memories), a phenomenon seen in children exposed to maltreatment-related trauma (Valentino et al, 2009) that may represent a neurocognitive risk marker linked to increased vulnerability to later psychiatric disorders (Rawal and Rice, 2012).

As hypothesized, decreased activation in the cerebellar vermis and right cerebellum in the high- relative to the lowtrauma-exposed group was observed during neutral/relaxing trials. Cerebellar regions are also notable for their high density of glucocorticoid receptors (Pavlik and Buresova, 1984). Relaxation aided by biofeedback has been found to elicit activation in the cerebellar vermis (Critchley et al, 2001). Given the suggested role of the cerebellar vermis as part of a network to detect and regulate autonomic arousal and the role of the cerebellum in processing affect (Critchley et al, 2000), hypofunctionality of this region could reflect the diminished self-regulatory control that has previously been described in individuals exposed to abusive or neglecting environments (Crittenden and Ainsworth, 1989). The role of cerebellar structures in affective situations remains largely unexplored and warrants further study. The anticipated hypofunctionality of the ACC during relaxation in the high-trauma group was not observed. This may relate to the different relaxation procedures used in comparison to previous studies (eg, imagery vs volitional control) (Critchley et al, 2001).

Relaxation training may be useful for tackling sequelae of trauma such as stress hyper-responsivity and depression (Grossman et al, 2004) and could supplement traditional psychotherapeutic and pharmacological treatments. The findings indicate relaxing imagery is processed differently in high- relative to low-trauma-exposed adolescents. Future research could explore if this reflects differential capacities of trauma-exposed individuals to benefit from relaxationbased interventions. It would be of further interest to assess whether physiological and neurobiological responses to the dynamic process of relaxation (the relaxation response) following emotional events are linked to trauma exposure.

Rates of obesity and associated health risks (eg, type II diabetes) are significantly elevated in those with a history of abuse (Thomas et al, 2008), even when relevant confounds have been controlled (Bentley and Widom, 2009). Contrary to our predictions, we found no differences between the high- and low-trauma groups in response to the favoritefood cues. This suggests that the association between early adversity and obesity-related outcomes is unlikely to be easily explained in terms of an individual's appetitive responses to the favorite-food cues. One possibility is that differences in appetitive response may be modulated by context. In other words, heightened stress reactivity in the trauma group may serve to amplify appetitive drive only under conditions of stress; manipulating the presence of food cues in the presence or absence of stress is required to test this possibility. Alternatively, it may be that traumaexposed individuals' responses to different types of food than those studied here or the additional cues present in real-life food-exposure situations account for the lack of observed differences. Data also indicate that stressrelated hormones and chronic inflammatory responses may contribute to obesity and diabetes (Black, 2003; Danese et al. 2009). Finally, obesity relating to trauma may be mediated by psychiatric disorders subsequent to 
maltreatment (Blaine, 2008). These possibilities warrant direct examination.

\section{Limitations and Future Directions}

As an initial study to assess responses to multiple emotional/motivational cues in youth stratified by traumaexposure levels, some variables that may prove informative in later studies were not assessed. Broad assessments of adversity throughout childhood could be supplemented by assessing the impact of trauma exposure during circumscribed periods in future studies. Abusive experiences at particular ages could have differential effects on brain regions and behavioral sequelae (Andersen et al, 2008). Gender may also modulate consequences of different early experiences, but was not investigated because of the sample size constraints (Thompson et al, 2004). These considerations should be investigated in future studies.

A potential confound associated with studying a disadvantaged population included the presence of prenatal cocaine exposure, which was associated with traumaexposure status. Our secondary analyses suggested that cocaine exposure did not have a strong impact on the observed results. However, the co-occurrence of trauma and cocaine exposure does mean that some variance in results could still be partly attributable to effects of prenatal cocaine. In a matched sample of prenatally cocaine-exposed and non-drug-exposed adolescents in this cohort, prenatal cocaine exposure was associated with blunted activation of the striatum and prefrontal cortex during the favorite-foodcue condition and increased occipital activation during the neutral-relaxing condition (Yip et al, 2014). These findings are at least suggestive that there are dissociable neural contributions from exposure to cocaine prenatally and childhood maltreatment. However, further research to disentangle the contributions of prenatal drug exposure and later trauma is warranted. One further caveat was that 'low trauma' defined relative to individuals in a disadvantaged population may not reflect low trauma more generally. The presence of childhood adversity even in the low-trauma group population may have obscured a main effect of trauma that would be evident comparing a veryhigh trauma group with individuals who have not been exposed to any trauma.

There are also limitations related to use of a crosssectional design. Firstly, causal relationships between trauma and later brain responses cannot be determined given factors such as gene-environment effects. However, animal research suggests causal roles of traumatic experiences on brain structure and function that likely translate to humans (Pryce et al, 2005). Secondly, to ascertain whether the differential activity observed in each trauma group represents neural biomarkers for later psychiatric disturbance, this cross-sectional study should be followed up with assessments as to whether patterns of brain activity mediate the relationship between maltreatment and later outcomes. This longitudinal cohort is well suited to this task.

The representativeness of this sample should be considered when extrapolating to other trauma-exposed individuals. First, some effects of adversity may be tempered or otherwise affected by being monitored by researchers every 6 months since birth. The sample was also of predominantly African-American descent, which may impact the generalizability of these findings to other ethnic groups. The sample also had lower than average IQs, and some individuals had been prenatally exposed to cocaine, which is not the case in all instances of childhood trauma. Our findings expand upon previous maltreatment research by investigating a wider spectrum of personalized cues at a crucial developmental stage. The current findings suggest possible neurobiological substrates that are impacted by childhood trauma and might relate to emotional and behavioral difficulties later in life.

\section{FUNDING AND DISCLOSURE}

The authors report that they have no financial conflicts of interest with respect to the content of this manuscript. Dr Sinha is on the Scientific Advisory Board for Embera Neurotherapeutics and Arkeo Pharmaceuticals. Dr Potenza has received financial support or compensation for the following: Dr Potenza has consulted for Ironwood, Lundbeck and Shire; has received research support from Mohegan Sun Casino, the National Center for Responsible Gaming, and Psyadon pharmaceuticals; has participated in surveys, mailings, or telephone consultations related to drug addiction, impulse control disorders, or other health topics; has consulted for law offices and the federal public defender's office in issues related to impulse control disorders; provides clinical care in the Connecticut Department of Mental Health and Addiction Services Problem Gambling Services Program; has performed grant reviews for the National Institutes of Health and other agencies; has guest-edited journal sections; has given academic lectures in grand rounds, CME events and other clinical or scientific venues; and has generated books or book chapters for publishers of mental health texts.

\section{ACKNOWLEDGMENTS}

We thank Matthew Freiburger and Ann Thomasson for their considerable assistance in overseeing data collection, and Kathy Armstrong, Iris Balodis, and Keri Bergquist for their assistance with imagery script development. This research was supported by the National Institutes of Health grants P50 DA016556, UL1-DE19586, RL1 AA017539, R01 DA006025, R01 DA017863 and K05 DA020091, the Office of Research on Women's Health, and the NIH Roadmap for Medical Research/Common Fund. The contents are solely the responsibility of the authors and do not necessarily represent the official views of any of the other funding agencies.

\section{REFERENCES}

Andersen SL, Tomada A, Vincow ES, Valente E, Polcari A, Teicher MH (2008). Preliminary evidence for sensitive periods in the effect of childhood sexual abuse on regional brain development. J Neuropsychiatry Clin Neurosci 20: 292-301.

Anderson CM, Teicher MH, Polcari A, Renshaw PF (2002). Abnormal T2 relaxation time in the cerebellar vermis of adults sexually abused in childhood: potential role of the vermis in stress-enhanced risk for drug abuse. Psychoneuroendocrinology 27: $231-244$. 
Bentley T, Widom CS (2009). A 30-year follow-up of the effects of child abuse and neglect on obesity in adulthood. Obesity 17: 1900-1905.

Bernstein DP, Ahluvalia T, Pogge D, Handelsman L (1997). Validity of the Childhood Trauma Questionnaire in an adolescent psychiatric population. J Am Acad Child Adolesc Psychiatry 36: 340-348.

Bernstein DP, Fink L (1998). Childhood Trauma Questionnaire: A retrospective self-report manual. The Psychological Corporation: San Antonio, TX, USA.

Black PH (2003). The inflammatory response is an integral part of the stress response: implications for atherosclerosis, insulin resistance, type II diabetes and metabolic syndrome X. Brain Behav Immun 17: 350-364.

Blaine B (2008). Does depression cause obesity? A meta-analysis of longitudinal studies of depression and weight control. $J$ Health Psychol 13: 1190-1197.

Cohen P, Brown J, Smaile E (2001). Child abuse and neglect and the development of mental disorders in the general population. Dev Psychopathol 13: 981-999.

Cohen S (1988). Perceived stress in a probability sample of the United States. In: Spacapan S, Oskamp S (eds) The social psychology of health. The Claremont Symposium on Applied Psychology. Sage: Tousand Oaks, CA, pp 31-67.

Cox RW (1996). AFNI: software for analysis and visualization of functional magnetic resonance neuroimages. Comput Biomed Res 29: 162-173.

Critchley HD, Elliott R, Mathias CJ, Dolan RJ (2000). Neural activity relating to generation and representation of galvanic skin conductance responses: a functional magnetic resonance imaging study. J Neurosci 20: 3033-3040.

Critchley HD, Melmed RN, Featherstone E, Mathias CJ, Dolan RJ (2001). Brain activity during biofeedback relaxation: a functional neuroimaging investigation. Brain 124: 1003-1012.

Critchley HD, Wiens S, Rotshtein P, Ohman A, Dolan RJ (2004). Neural systems supporting interoceptive awareness. Nat Neurosci 7: 189-195.

Crittenden DM, Ainsworth MD (1989). Child maltreatment and attachment theory. In: Cicchetti D (ed). Child Maltreatment: Theory and research on the causes and consequences of child abuse and neglect. Cambridge University Press: Cambridge, pp 432-463.

Danese A, Moffitt TE, Harrington H, Milne BJ, Polanczyk G, Pariante CM et al (2009). Adverse childhood experiences and adult risk factors for age-related disease: depression, inflammation, and clustering of metabolic risk markers. Arch Pediatr Adolesc Med 163: 1135-1143.

Dannlowski U, Stuhrmann A, Beutelmann V, Zwanzger P, Lenzen T, Grotegerd D et al (2012). Limbic scars: long-term consequences of childhood maltreatment revealed by functional and structural magnetic resonance imaging. Biol Psychiatry 71: 286-293.

Dolcos F, LaBar KS, Cabeza R (2005). Remembering one year later: role of the amygdala and the medial temporal lobe memory system in retrieving emotional memories. Proc Natl Acad Sci USA 102: 2626-2631.

Duncan JS, Papademetris X, Yang J, Jackowski M, Zeng X, Staib LH (2004). Geometric strategies for neuroanatomic analysis from MRI. NeuroImage 23: S34-S45.

Edmiston EE, Wang F, Mazure CM, Guiney J, Sinha R, Mayes LC et al (2011). Corticostriatal-limbic gray matter morphology in adolescents with self-reported exposure to childhood maltreatment. Arch PediatrAdolesc Med 165: 1069-1077.

Fink LA, Bernstein D, Handelsman L, Foote J, Lovejoy M (1995). Initial reliability and validity of the childhood trauma interview: a new multidimensional measure of childhood interpersonal trauma. Am J Psychiatry 152: 1329-1335.

Fisher RA (1921). On the "probable error" of a coefficient of correlation deduced from a small sample. Metron 1: 3-32.
Gilbert R, Widom CS, Browne K, Fergusson D, Webb E, Janson S (2009). Burden and consequences of child maltreatment in high-income countries. Lancet 373: 68-81.

Grossman P, Niemann L, Schmidt S, Walach H (2004). Mindfulness-based stress reduction and health benefits. A metaanalysis. J Psychosom Res 57: 35-43.

Heim C, Nemeroff CB (2001). The role of childhood trauma in the neurobiology of mood and anxiety disorders: preclinical and clinical studies. Biol Psychiatry 49: 1023-1039.

Holmes CJ, Hoge R, Collins L, Woods R, Toga AW, Evans AC (1998). Enhancement of MR images using registration for signal averaging. J Comput Assist Tomogr 22: 324-333.

Hommer RE, Seo D, Lacadie CM, Chaplin TM, Mayes LC, Sinha R et al (2013). Neural correlates of stress and favorite-food cue exposure in adolescents: a functional magnetic resonance imaging study. Hum Brain Mapp 34: 2561-2573.

Jastreboff AM, Sinha R, Lacadie C, Small DM, Sherwin RS, Potenza MN (2013). Neural correlates of stress- and food cueinduced food craving in obesity: association with insulin levels. Diabetes Care 36: 394-402.

Jeffry A, Belsky J (2008). Attachment theory within a modern evolutionary framework. In: Cassidy J, Shaver PR (eds). Handbook of attachment: Theory, research, and clinical applications, 2nd ed. Guilford Press: New York, NY, pp 131-157.

Kaufman AS, Kaufman NL (1983). Kaufman assessment battery for children. American Guidance Service: Circle Pines, MN, USA.

Kelly PA, Viding E, Wallace GL, Schaer M, De Brito SA, Robustelli B et al (2013). Cortical thickness, surface area, and gyrification abnormalities in children exposed to maltreatment: neural markers of vulnerability? Biol Psychiatry 74: 845-852.

Loman MM, Gunnar MR (2010). Early experience and the development of stress reactivity and regulation in children. Neurosci Biobehav Rev 34: 867-876.

Mayes LC, Molfese DL, Key AP, Hunter NC (2005). Event-related potentials in cocaine-exposed children during a Stroop task. Neurotoxicol Teratol 27: 797-813.

McCrory EJ, De Brito SA, Sebastian CL, Mechelli A, Bird G, Kelly PA et al (2011). Heightened neural reactivity to threat in child victims of family violence. Curr Biol 21: R947-R948.

Nolf EX, Voet T, Jacobs F, Dierckx R, Lemahieu IX (2003). An open-source medical image conversion toolkit. Eur J Nucl Med 30: S246.

Ochsner KN, Bunge SA, Gross JJ, Gabrieli JD (2002). Rethinking feelings: an FMRI study of the cognitive regulation of emotion. $J$ Cogn Neurosci 14: 1215-1229.

Paivio SC, Cramer KM (2004). Factor structure and reliability of the childhood trauma questionnaire in a Canadian undergraduate student sample. Child Abuse Negl 28: 889-904.

Paus T, Keshavan M, Giedd JN (2008). Why do many psychiatric disorders emerge during adolescence? Nat Rev Neurosci 9: 947-957.

Pavlik A, Buresova M (1984). The neonatal cerebellum: the highest level of glucocorticoid receptors in the brain. Brain Res 314: $13-20$.

Pollak SD (2008). Mechanisms linking early experience and the emergence of emotions: illustrations from the study of maltreated children. Curr Dir Psychol Sci 17: 370-375.

Pryce CR, Ruedi-Bettschen D, Dettling AC, Weston A, Russig H, Ferger B et al (2005). Long-term effects of early-life environmental manipulations in rodents and primates: potential animal models in depression research. Neurosci Biobehav Rev 29: 649-674.

Rawal A, Rice F (2012). Examining overgeneral autobiographical memory as a risk factor for adolescent depression. J Am Acad Child Adolesc Psychiatry 51: 518-527.

Sander D, Grafman J, Zalla T (2003). The human amygdala: an evolved system for relevance detection. Rev Neurosci 14: 303-316. 
Scher CD, Stein MB, Asmundson GJ, McCreary DR, Forde DR (2001). The childhood trauma questionnaire in a community sample: psychometric properties and normative data. J Trauma Stress 14: 843-857.

Seo D, Tsou KA, Ansell EB, Potenza MN, Sinha R (2014). Cumulative adversity sensitizes neural response to acute stress: association with health symptoms. Neuropsychopharmacology 39: $670-680$

Shaffer D, Fisher P, Lucas CP, Dulcan MK, Schwab-Stone ME (2000). NIMH Diagnostic Interview Schedule for Children Version IV (NIMH DISC-IV): description, differences from previous versions, and reliability of some common diagnoses. J Am Acad Child Adolesc Psychiatry 39: 28-38.

Sinha R (2009). Modleing stress and drug craving in the laboratory: implications for addiction treatment and development. Addict Biol 14: 84-98.

Sinha R (2013). Modeling relapse situations in the human laboratory. Curr Top Behav Neurosci 13: 379-402.

Sinha R, Tuit K (2012). Imagery Script Development Procedures Manual. CreateSpace Independent Publishing Platform: North Charleston.

Somerville LH, Jones RM, Casey BJ (2010). A time of change: behavioral and neural correlates of adolescent sensitivity to appetitive and aversive environmental cues. Brain $\operatorname{Cogn} 72$ : 124-133.

Thomas C, Hypponen E, Power C (2008). Obesity and type 2 diabetes risk in midadult life: the role of childhood adversity. Pediatrics 121: e1240-e1249.

Thompson MP, Kingree JB, Desai S (2004). Gender differences in longterm health consequences of physical abuse of children: data from a nationally representative survey. Am J Public Health 94: 599-604.

Valentino K, Toth SL, Cicchetti D (2009). Autobiographical memory functioning among abused, neglected, and nonmaltreated children: the overgeneral memory effect. J Child Psychol Psychiatry 50: 1029-1038.

Woon FL, Hedges DW (2008). Hippocampal and amygdala volumes in children and adults with childhood maltreatmentrelated posttraumatic stress disorder: a meta-analysis. Hippocampus 18: 729-736.

Xiong J, Gao JH, Lancaster JL, Fox PT (1995). Clustered pixels analysis for functional MRI activation studies of the human brain. Human Brain Mapp 3: 287-301.

Yip SW, Potenza EB, Balodis IM, Lacadie CM, Sinha R, Mayes LC et al (2014). Prenatal cocaine exposure and adolescent neural responses to appetitive and stressful stimuli. Neuropsychopharmacology 39: 2824-2834.

Supplementary Information accompanies the paper on the Neuropsychopharmacology website (http://www.nature.com/npp) 